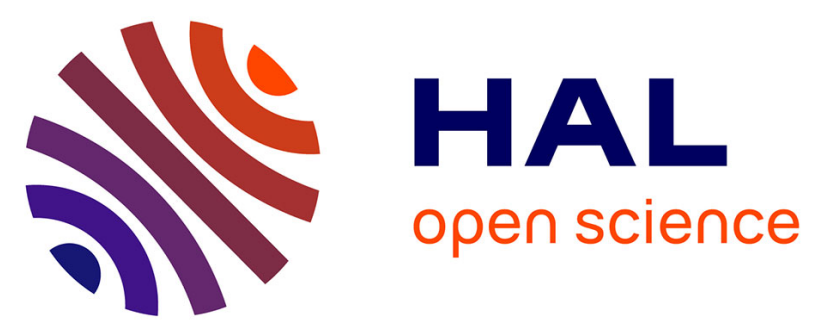

\title{
A decentralized multilayer sliding mode control architecture for vehicle's global chassis control, and comparison with a centralized architecture
}

\author{
Ali Hamdan, Abbas Chokor, Reine Talj, Moustapha Doumiati
}

\section{To cite this version:}

Ali Hamdan, Abbas Chokor, Reine Talj, Moustapha Doumiati. A decentralized multilayer sliding mode control architecture for vehicle's global chassis control, and comparison with a centralized architecture. 2nd International Conference on Electronic Engineering and Renewable Energy (ICEERE 2020), Apr 2020, Saidia, Morocco. pp.583-591. hal-02542144

HAL Id: hal-02542144

https://hal.science/hal-02542144

Submitted on 18 Dec 2020

HAL is a multi-disciplinary open access archive for the deposit and dissemination of scientific research documents, whether they are published or not. The documents may come from teaching and research institutions in France or abroad, or from public or private research centers.
L'archive ouverte pluridisciplinaire HAL, est destinée au dépôt et à la diffusion de documents scientifiques de niveau recherche, publiés ou non, émanant des établissements d'enseignement et de recherche français ou étrangers, des laboratoires publics ou privés. 


\title{
A decentralized multilayer sliding mode control architecture for vehicle's global chassis control, and comparison with a centralized architecture
}

\author{
Ali Hamdan ${ }^{1}$, Abbas Chokor $^{1}$, Reine Talj*1, and Moustapha Doumiati ${ }^{2}$ \\ 1 Sorbonne universités, Université de technologie de Compiègne, CNRS, Heudiasyc UMR \\ 7253, CS 60 319, 60203 Compiègne, France. \\ *reine.talj@hds. utc.fr \\ 2 ESEO-IREENA EA 4642, 10 Bd Jeanneteau, 49100 Angers, France.
}

\begin{abstract}
This paper presents a decentralized Global Chassis Control (GCC) architecture. The objective of this global chassis controller is to improve the overall vehicle performance i.e maneuverability, lateral stability and rollover avoidance, by coordinating the Active Front steering, Direct Yaw Control and Active Suspensions in a decentralized architecture. The developed architecture is multilayer, and based on higher order sliding-mode control, the super-twisting algorithm. The proposed GCC is validated by simulation using Matlab/Simulink, and a comparison is done with a centralized $L P V / \mathscr{H}_{\infty}$ architecture that has been developed in the laboratory, to show the difference in behavior and performance of both strategies of control.
\end{abstract}

Decentralized Multilayer Control Architecture; Global Chassis Control; Active Suspensions; Direct Yaw Control; Active Front Steering; Sliding Mode Control.

\section{INTRODUCTION}

Active safety is an important feature into the intelligent vehicles. According to the "National Highway Traffic Safety Administration (NHTSA)" statistics, human's faults cause almost 90\% of road accidents as explained in [1]. Advanced Driving Assistance System (ADAS) influences on the behavior of vehicle on the road, and helps the driver in the driving process in order to avoid a dangerous situation. ADAS systems are formed by several single-actuator approaches that have been proposed and marketed, such as: Electronic Stability Program (ESP) or Direct Yaw Control (DYC) to enhance the vehicle lateral stability; Active Front Steering (AFS) to mainly improve the vehicle maneuverability or lane keeping; and (Semi-) Active Suspensions (AS) to improve comfort, road holding and rollover avoidance [2].

Many advanced studies are developed in literature to improve the global performance of the vehicle in different driving situations. These studies suggest coordination between several ADAS systems known as Global Chassis Control (GCC). The GCC system deals with the complexity of control problems for Multi-Input-Multi-Output (MIMO) systems. The main idea of the GCC is the coordination between the AFS and the DYC to improve the vehicle maneuverability and lateral stability depending on the driving 
situation. Many advanced control approaches have been proposed for this issue. The authors in [3] applied a decentralized approach where they developed a DYC controller for lateral stability purpose and an AFS controller for maneuverability purpose, based on sliding mode technique, and then a monitor switches between the two controllers according to the driving situations. However, the overall stability of the system is not guaranteed in the decentralized approach, but it is simple to develop, implement and tune. In [4], [5], the authors propose several robust and optimal centralized controllers for the MIMO system based on the LPV/ $\mathscr{H}_{\infty}$ control technique, where the $L P V / \mathscr{H}_{\infty}$ controller penalizes or relaxes the steering and braking to enhance maneuverability and lateral stability. By using this method, the overall stability of the system is guaranteed and a polytopic approach is used to actuate the different controllers. However, these controllers were synthesized while disregarding the roll motion; the deduced rollover enhancement was a consequence of the lateral stability control. Authors in [6], [7] have presented several centralized $L P V / \mathscr{H}_{\infty}$ controllers, where AFS, DYC and AS are used to control the decoupled lateral and vertical vehicle dynamics. From the other side, authors in [8] and [9], have used the roll angle and its angular velocity to control the vehicle load transfer that leads to rollover avoidance. Moreover, authors deduced lateral stability improvement as a consequence of roll control. Centralized architectures are optimal in global performance, but are more complex to design and to implement, and could take an important amount of calculation.

All these interesting research have motivated us to study the control of the vehicle yaw rate, the side slip angle and the roll angle in order to improve the overall vehicle performance. Thus, in our present work, a decentralized multilayer control structure, based on sliding mode super-twisting control approach, is developed to improve the maneuverability, lateral stability, and rollover avoidance using steering, braking actuators and active suspension system. A comparison between the proposed controller and a centralized architecture presented in [10] is done. The paper structure is as follows: Section 2 exposes the extended bicycle model of the vehicle based on the combination of the coupled lateral (yaw and side-slip) and roll motions. In Section 3, the proposed decentralized control architecture is detailed. Simulation validation of the proposed approach is reported in Section 4. Finally, the conclusions and the perspectives of this work are given in Section 5.

\section{Vehicle model}

The vehicle is a group of interconnected mechanical and electrical systems that make the vehicle behavior nonlinear. The ADAS systems such as AFS (Active Front Steering), active suspensions, differential braking, etc, improve the vehicle's performance (lateral motion, yaw motion, rolling motion, etc.). A complete nonlinear vehicle model has been developed in [11]. However, this model is a nonlinear model that does not respond to the formulation of control problems. For this reason, a linear simplified LTI vehicle model is used to develop the GCC controller. It is an extended bicycle model, with coupled lateral/vertical dynamics. For vertical dynamics, the rolling motion is considered, for being the most critical for stabilization problems and rollover avoidance. Hence, this LTI model is a coupled yaw-lateral-roll linear vehicle model, inspired from 
literature [8], and is given by the following equations of "Plant P":

$$
\text { PlantP }:\left\{\begin{array}{c}
I_{z} \ddot{\psi}=F_{y f} l_{f}+F_{y r} l_{r}+I_{x z} \ddot{\theta}+M_{z}+M_{d, \dot{\psi}}, \\
M \mathrm{~V}(\dot{\beta}+\dot{\psi})=F_{y f}+F_{y r}+M_{s} h_{\theta} \ddot{\theta}+F_{d, y}, \\
\left(I_{x}+M_{s} h_{\theta}^{2}\right) \ddot{\theta}=M_{s} h_{\theta} \mathrm{V}(\dot{\beta}+\dot{\psi})+\left(M_{s} g h_{\theta}-K_{\theta}\right) \theta \\
\quad-C_{\theta} \dot{\theta}+M_{d, \theta},
\end{array}\right.
$$

where the vehicle parameters and variables are given in Table $1 . F_{y f}$ represents the lateral force of the front left and right tires merged together at the center of the front axle. Similarly, $F_{y r}$ is noted for the rear axle. $F_{y f}$ and $F_{y r}$ are given as:

$$
\begin{aligned}
& F_{y f}=\mu C_{f} \alpha_{f}, \\
& F_{y r}=\mu C_{r} \alpha_{r},
\end{aligned}
$$

and the tires slip angles as:

$$
\begin{aligned}
& \alpha_{f}=-\beta-\frac{l_{f} \dot{\psi}}{V}+\delta_{t}, \\
& \alpha_{r}=-\beta+\frac{l_{r} \dot{\psi}}{V} .
\end{aligned}
$$

The reference "bicycle model" used in the control layer is presented in [1] and is given in (4):

$$
\left(\begin{array}{c}
\ddot{\psi}_{r e f} \\
\dot{\beta}_{r e f}
\end{array}\right)=\left[\begin{array}{c}
-\mu \frac{l_{f}^{2} c_{f}+l_{r}^{2} c_{r}}{I_{I_{x}} V_{x}} \mu \frac{l_{r} c_{r}-l_{f} c_{f}}{I_{z}} \\
-1+\mu \frac{I_{r} c_{r}-l_{f} c_{f}}{M V_{x}^{2}}-\mu \frac{c_{f}+c_{r}}{M V_{x}}
\end{array}\right]\left(\begin{array}{c}
\dot{\psi}_{r e f} \\
\beta_{r e f}
\end{array}\right)+\left[\begin{array}{c}
\mu \frac{l_{f} c_{f}}{I_{z_{f}}} \\
\mu \frac{\mathcal{c}_{f}}{M V_{x}}
\end{array}\right] \delta_{d},
$$

where $\delta_{d}$ is the driver steer angle on the front wheels, $\dot{\psi}_{\text {ref }}$ is the desired reference yaw rate, $\beta_{r e f}$ is the corresponding side slip angle, and $V_{x}$ is the vehicle longitudinal speed. For security reasons, the authors in [1] propose to saturate $\beta_{\text {ref }}$ and $\dot{\psi}_{\text {ref }}$ below a threshold, as described in (5):

$$
\begin{gathered}
\left|\dot{\psi}_{\text {ref }}\right| \leq\left|\frac{0.85 \mu g}{V_{x}}\right| \\
\beta_{\text {ref }}=\arctan (0.02 \mu g)
\end{gathered}
$$

\section{DECENTRALIZED GLOBAL CHASSIS CONTROL ARCHITECTURE}

The global decentralized multilayer control architecture of Fig. 1 is presented in this section. The output variables i.e the vehicle yaw rate $\psi$, the side-slip angle $\beta$, and the suspended mass roll $\theta$ are controlled independently by using the single-input, singleoutput controller based on the Super-Twisting Sliding Mode (STSM) technique. Let us introduce an overview of the theory of Super-Twisting Sliding Mode. The STSM is a robust control technique that forces the states of the system to reach a sliding surface during a finite time (convergence phase) and to stay on this surface (sliding phase) in presence of perturbations.

Consider the second order system given as:

$$
\ddot{x}=f(X, t)+g(X, t) u(t)
$$


A. Hamdan et al.

Table 1. Parameters Values for Simulation

\begin{tabular}{ccc} 
Symbols & Description & Parameters values \\
\hline$\dot{\psi}$ & Vehicle yaw rate & {$[\mathrm{rad} / \mathrm{s}]$} \\
$\beta$ & Vehicle side slip angle at CG & {$[\mathrm{rad}]$} \\
$\theta$ & Sprung mass roll angle & {$[\mathrm{rad}]$} \\
$F_{y_{i}}$ & Lateral forces at the $i$ axle & {$[\mathrm{N}]$} \\
$\delta_{d}$ & Driver steering angle & {$[\mathrm{rad}]$} \\
$V$ & Vehicle speed & {$[\mathrm{m} / \mathrm{s}]$} \\
$I_{x}$ & Roll moment of inertia of sprung mass & $534\left[\mathrm{~kg} . \mathrm{m}^{2}\right]$ \\
$I_{z}$ & Vehicle yaw moment of inertia & $1970\left[\mathrm{~kg} . \mathrm{m}^{2}\right]$ \\
$I_{x z}$ & Vehicle yaw-roll product of inertia & $743\left[\mathrm{~kg} . \mathrm{m}^{2}\right]$ \\
$t_{f}$ & Half front track & $0.773[\mathrm{~m}]$ \\
$t_{r}$ & Half rear track & $0.773[\mathrm{~m}]$ \\
$l_{f}$ & Wheelbase to the front & $1.0385[\mathrm{~m}]$ \\
$l_{r}$ & Wheelbase to the rear & $1.6015[\mathrm{~m}]$ \\
$h_{\theta}$ & Sprung mass roll arm & $0.27[\mathrm{~m}]$ \\
$M_{s}$ & Sprung mass & $1126.4[\mathrm{~kg}]$ \\
$C_{f}, C_{r}$ & Front, rear tire cornering stiffness & $76776[\mathrm{~N} / \mathrm{rad}]$ \\
$K_{\theta}$ & Rolling suspension angular stiffness & $30000[\mathrm{~N} . \mathrm{m} / \mathrm{s}]$ \\
$C_{\theta}$ & Rolling suspension angular damper & $10000[\mathrm{~N} . \mathrm{m} / \mathrm{s}]$ \\
$g$ & Gravity constant & $9.81\left[\mathrm{~m} / \mathrm{s}^{2}\right]$ \\
$\mu$ & Road adherence coefficient & dry surface=1 $[-]$ \\
\hline
\end{tabular}

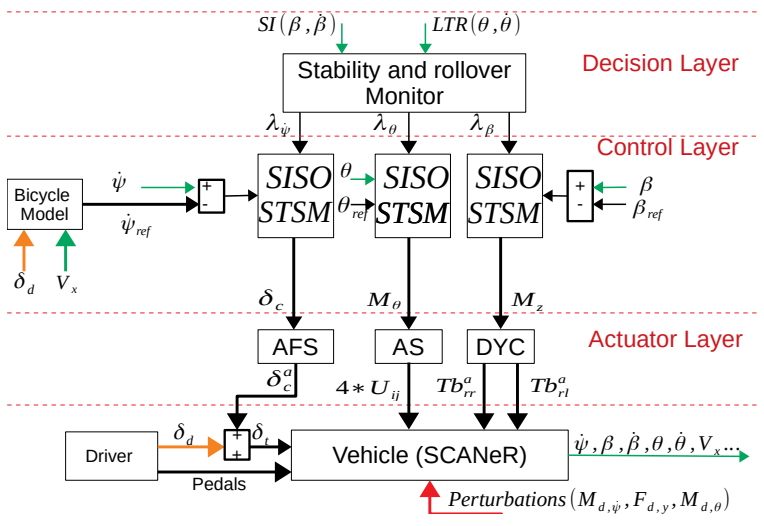

Fig. 1. Decentralized global chassis control architecture 
where $X=[x, \dot{x}]^{T} \in \mathfrak{R}^{2}$ is the state vector, $u$ is the control input, and $f, g$ are continuous functions. $X_{\text {des }}$ is the desired state of $X$ with $X_{\text {des }}=\left[x_{d e s}, \dot{x}_{d e s}\right]^{T} \in \mathfrak{R}^{2}$. The error vector is given by $E=X-X_{d e s}=[e, \dot{e}]^{T} \in \mathfrak{R}^{2}$ where $e=x-x_{d e s}$ and $\dot{e}=\dot{x}-\dot{x}_{d e s}$. Therefore, a sliding variable $s$ with relative degree $r=1$ w.r.t the control input, is defined as:

$$
s=\dot{e}+k e,
$$

The second order derivative of $s$ is:

$$
\ddot{s}(s, t)=\Phi(s, t)+\xi(s, t) \dot{u}(t)
$$

where $\Phi(s, t)$ and $\xi(s, t)$ are the unknown bounded signals.

The goal of the Super-Twisting algorithm is to enforce the sliding variable $s$ to converge to zero $(s=0)$ in finite time. Assume that there exist positive constants $S_{0}, b_{\min }, b_{\max }$, $C_{0}, U_{\max }$ verifying for all $x \in \mathfrak{R}^{n}$ and $|s(x, t)|<S_{0}$ :

$$
\left\{\begin{array}{l}
|u(t)| \leq U_{\max } \\
|\Phi(s, t)|<C_{0} \\
0<b_{\min } \leq|\xi(s, t)| \leq b_{\max }
\end{array}\right.
$$

Thus, the control input based on the Super-Twisting Sliding Mode algorithm, is given as:

$$
u(t)=u_{1}+u_{2}\left\{\begin{array}{l}
\left.\left.u_{1}=-\alpha_{1}|s|^{\tau} \operatorname{sign}(s), \quad \tau \in\right] 0,0.5\right] \\
\dot{u}_{2}=-\alpha_{2} \operatorname{sign}(s)
\end{array}\right.
$$

$\alpha_{1}$ and $\alpha_{2}$ are positive gains. The following conditions guarantee the finite time convergence:

$$
\left\{\begin{array}{l}
\alpha_{1} \geq \sqrt{\frac{4 C_{0}\left(b_{\max } \alpha_{2}+C_{0}\right)}{b_{\min }^{2}\left(b_{\min } \alpha_{2}-C_{0}\right)}} \\
\alpha_{2}>\frac{C_{0}}{b_{\min }}
\end{array}\right.
$$

The analysis of convergence is presented in [12]. An approximation function $\frac{s}{|s|+\varepsilon}$ is used to smooth the $\operatorname{sign}(s)$ function, where $\varepsilon>0$.

Let us define the three sliding variables for the three decentralized controllers as follows:

$$
\begin{aligned}
& s_{\dot{\psi}}=e_{\dot{\psi}}=\dot{\psi}-\dot{\psi}_{r e f}, \\
& s_{\beta}=e_{\beta}=\beta-\beta_{r e f}, \\
& s_{\theta}=\dot{e}_{\theta}+k_{\theta} e_{\theta}=\left(\dot{\theta}-\dot{\theta}_{r e f}\right)+k_{\theta}\left(\theta-\theta_{r e f}\right),
\end{aligned}
$$

The sliding variables $s_{\dot{\psi}}, s_{\beta}$ and $s_{\theta}$ have a relative degree equal to one w.r.t $\delta_{c}, M_{z}$ and $M_{\theta}$ respectively. Thus, in order to converge these variables to zero and the controlled states follow the desired ones, and based on the above discussion, the control inputs of AFS, DYC and AS applied to the system, are given by:

$$
\begin{aligned}
& \delta_{c}=-\alpha_{\delta, 1}\left|s_{\dot{\psi}}\right|^{\tau_{\delta}} \operatorname{sign}\left(s_{\dot{\psi}}\right)-\alpha_{\delta, 2} \int_{0}^{t} \operatorname{sign}\left(s_{\dot{\psi}}\right) d \tau, \\
& M_{z}=-\alpha_{M_{z}, 1}\left|s_{\beta}\right| \tau_{M_{z}} \operatorname{sign}\left(s_{\beta}\right)-\alpha_{M_{z}, 2} \int_{0}^{t} \operatorname{sign}\left(s_{\beta}\right) d \tau \\
& M_{\theta}=-\alpha_{M_{\theta}, 1}\left|s_{\theta}\right|^{\tau_{M_{\theta}}} \operatorname{sign}\left(s_{\theta}\right)-\alpha_{M_{\theta}, 2} \int_{0}^{t} \operatorname{sign}\left(s_{\theta}\right) d \tau \text {, }
\end{aligned}
$$

where $\alpha_{\delta, i}, \alpha_{M_{z}, i}$ and $\alpha_{M_{\theta, i}}$ with $i=[1,2]$, are positive constants satisfying the conditions in (11). $\tau_{\delta}, \tau_{M_{z}}$ and $\tau_{M_{\theta}}$ are constants between $\left.] 0,0.5\right]$. 
The decision layer monitors the driving situation based on SI (lateral stability index) and LTR (load transfer ratio) criteria, then it delivers the different gains $\lambda_{i}$ in order to activate or deactivate the different actuators. These gains are given as follows:

$$
\begin{gathered}
\lambda_{\beta}=\frac{1}{1+e^{-\frac{8}{S I}-\underline{S I}}\left(S I-\frac{\overline{S I}+\underline{S I}}{2}\right)}, \\
\lambda_{\psi}=1-\lambda_{\beta} . \\
\lambda_{\theta}=\frac{1}{1+e^{-\frac{8}{L T R-\underline{L T R}}\left(L T R-\frac{\overline{L T R}+\underline{L T R}}{2}\right)},}
\end{gathered}
$$

\section{SIMULATION RESULTS}

In this section, the developed controller will be validated with a double lane change test at $110 \mathrm{~km} / \mathrm{h}$ as initial speed. All simulations are done using Matlab/Simulink with a complete nonlinear model of the vehicle [11], validated on "SCANeR Studio" (OKtal $)^{3}[13]$. Then, a comparison is done between an uncontrolled vehicle, where no controller is used ("OL" as Open Loop) and controlled vehicle equipped with two different controllers, i.e, the decentralized controller ("STSM" as Super-Twisting Sliding Mode) and a centralized controller (" $L P V / \mathscr{H}_{\infty}$ ") developed in the laboratory Heudiasyc, and presented in [10]. During this test, The driver's intention is to change the lane in a short time and then return to the same lane. Noting that in the two techniques of control, the active suspensions system AS aims to avoid rollover by decreasing the angle $\theta$.

Fig. 2, 3 and 4 show the different control variables such as the yaw rate, the side-slip

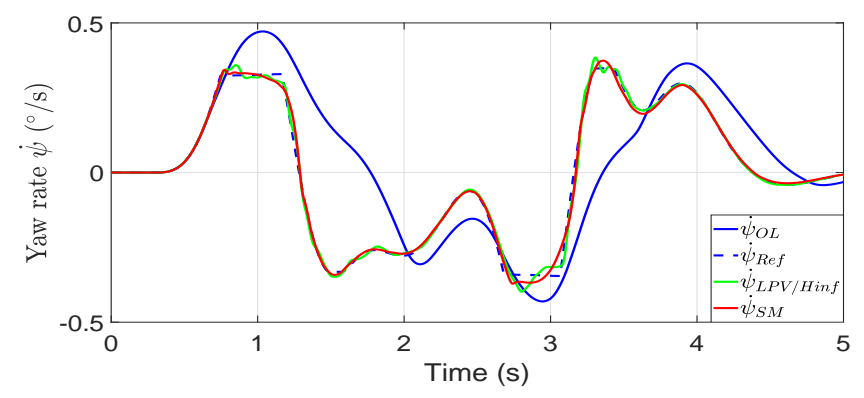

Fig. 2. Yaw rate comparison

angle and the roll angle respectively. Fig. 2 shows that the yaw rate tracks the reference yaw rate delivered by the bicycle model, and both controllers have almost the same behavior compared with the uncontrolled vehicle. Thus, the maneuverability objective is achieved. In order to improve the lateral stability and to prevent an undesirable driver

3 "SCANeR Studio" is a simulator dedicated to vehicle dynamics simulations. 


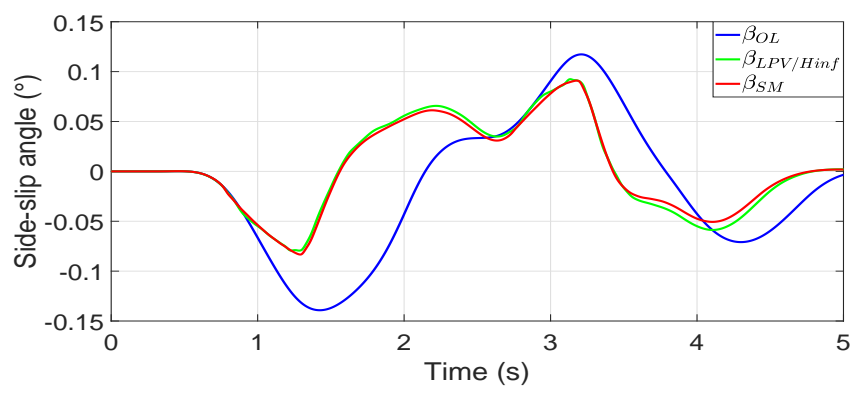

Fig. 3. Side-slip angle comparison

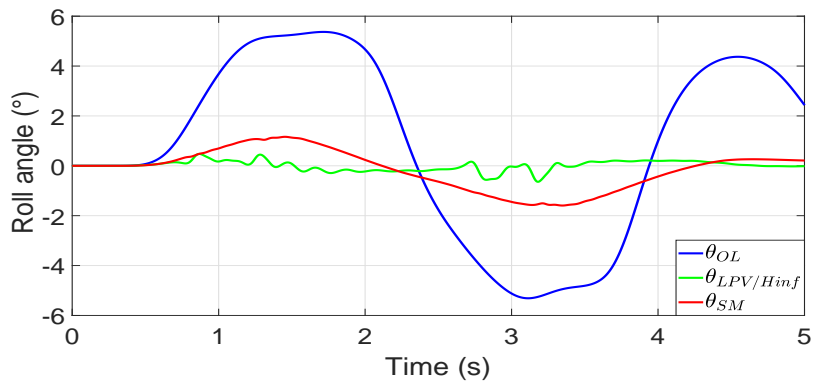

Fig. 4. Roll angle comparison

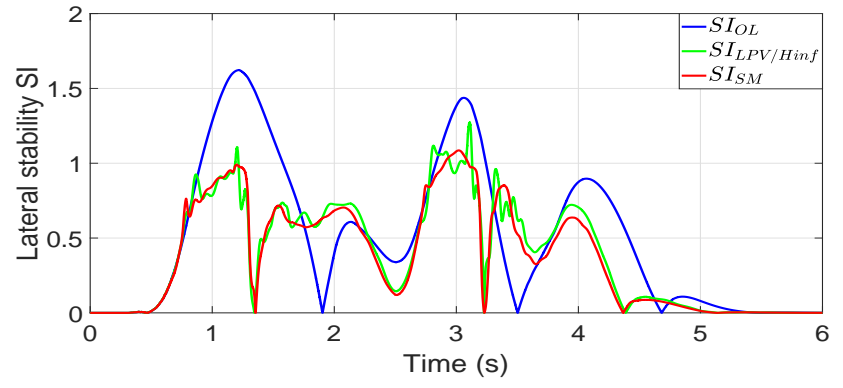

Fig. 5. Lateral stability index comparison 


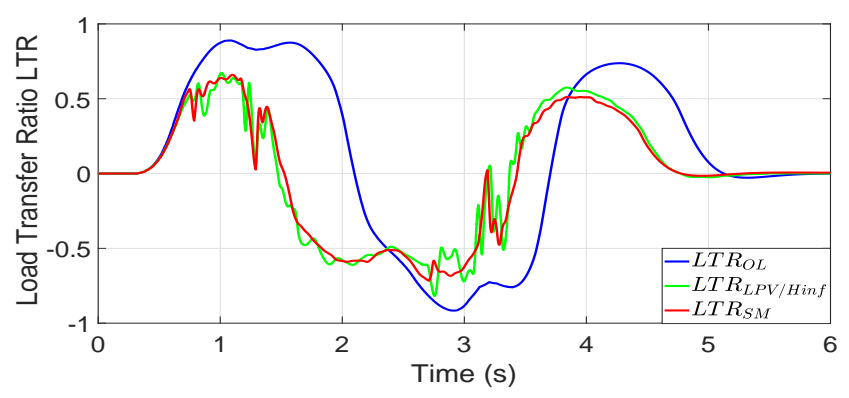

Fig. 6. Load Transfer Ratio comparison

situation, the side-slip angle should be reduced as shown in Fig. 3. Both control architectures have similar influence on this angle. On the other hand, the convergence of roll angle to zero allows the avoidance of rollover risk, by reducing the load transfer ratio $L T R$. The Fig. 4 shows that the $L P V / \mathscr{H}_{\infty}$ controller is capable to diminish more the roll angle to zero compared to the STSM controller.

Fig. 5 shows the lateral stability index (SI), and Fig. 6 presents the lateral load transfer ratio (LTR). Both SI and LTR are improved with both GCC architectures. Hence, lateral stability and rollover avoidance are enhanced.

\section{CONCLUSION AND PERSPECTIVES}

To conclude, a decentralized multilayer sliding mode control architecture has been developed to improve the overall vehicle performance. This enhancement is done by coordination of the Active Front Steering, Direct Yaw Control and Active Suspensions in a decentralized controller. The proposed controller is validated by using Matlab/Simulink and a comparison is done with a centralized approach based on the $L P V / \mathscr{H}_{\infty}$ technique, presented in [10]. Results show an almost similar performance of the decentralized scheme with its centralized equivalent. However, decentralized architecture is simpler and easier to tune and implement than centralized controller. Hence, the decentralized architecture could be much more interesting. In future works, we will work on the proof of the global stability of the system with the decentralized global chassis controller, a Lyapunov-based analysis will be done to show the convergence and boundedness of the solution. Validation on the Scaner Studio simulator and on a real vehicle platform will also be investigated. In addition, other performance index will be also used to do the benchmark comparing against the other conventional control approaches.

Acknowledgement The authors would like to thank the Hauts-de-France Region and the European Regional Development Fund (ERDF) 2014/2020 for the funding of this work, through the SYSCOVI project. This work was also carried out in the framework of the Labex MS2T, (Reference ANR-11-IDEX-0004-02) and the Equipex ROBOTEX (Reference ANR-10-EQPX-44-01) which were funded by the French Government, through the program "Investments for the future" managed by the National Agency for Research. 


\section{Bibliography}

[1] Rajesh Rajamani. Vehicle Dynamics and Control. Springer, 2012.

[2] A Chokor, R Talj, M Doumiati, and A Charara. A global chassis control system involving active suspensions, direct yaw control and active front steering. IFAC-

PapersOnLine, 52(5):444-451, 2019.

[3] Junjie He, David A Crolla, MC Levesley, and WJ Manning. Coordination of active steering, driveline, and braking for integrated vehicle dynamics control. Proceedings of the Institution of Mechanical Engineers, Part D: Journal of Automobile Engineering, 220(10):1401-1420, 2006.

[4] Charles Poussot-Vassal, Olivier Sename, and Luc Dugard. Robust vehicle dynamic stability controller involving steering and braking systems. In IEEE European Control Conference (ECC), pages 3646-3651, 2009.

[5] Moustapha Doumiati, Olivier Sename, Luc Dugard, John-Jairo Martinez-Molina, Peter Gaspar, and Zoltan Szabo. Integrated vehicle dynamics control via coordination of active front steering and rear braking. European Journal of Control, 19(2):121-143, 2013.

[6] Olivier Sename, Peter Gaspar, and József Bokor. Robust control and linear parameter varying approaches: application to vehicle dynamics. Springer, vol. 437, 2013.

[7] Wuwei Chen, Hansong Xiao, Qidong Wang, Linfeng Zhao, and Maofei Zhu. Integrated vehicle dynamics and control. John Wiley \& Sons, 2016.

[8] Van Tan Vu, Olivier Sename, Luc Dugard, and Péter Gáspár. Enhancing roll stability of heavy vehicle by lqr active anti-roll bar control using electronic servo-valve hydraulic actuators. Vehicle System Dynamics, 55(9):1405-1429, 2017.

[9] Jialing Yao, Guangwei Lv, Min Qv, Zhihong Li, Shan Ren, and Saied Taheri. Lateral stability control based on the roll moment distribution using a semiactive suspension. Proceedings of the Institution of Mechanical Engineers, Part D: Journal of Automobile Engineering, 231(12):1627-1639, 2017.

[10] Abbas Chokor, Moustapha Doumiati, Reine Talj, and Ali Charara. Design of a new gain-scheduled lpv/ $h_{\infty}$ controller for vehicle's global chassis control. In 58th Conference on Decision and Control (CDC), 2019.

[11] Abbas Chokor, Reine Talj, Ali Charara, Hassan Shraim, and Clovis Francis. Active suspension control to improve passengers comfort and vehicle's stability. In 19th International Conference on Intelligent Transportation Systems (ITSC), pages 296-301. IEEE, 2016.

[12] V. Utkin. On convergence time and disturbance rejection of super-twisting control. IEEE Transactions on Automatic Control, 58(8):2013-2017, 2013.

[13] Abbas Chokor, Reine Talj, Ali Charara, Moustapha Doumiati, and Abdelhamid Rabhi. Rollover prevention using active suspension system. In 20th International Conference on Intelligent Transportation Systems (ITSC), pages 17061711. IEEE, 2017. 